José Luis Cabrera, Complejidades conceptuales sobre el colonialismo y lo postcolonial. Aproximaciones desde el caso del Pueblo Mapuche / Conceptual complexities of colonialism and postcolonial. Approaches from the case of the Mapuche people, Revista Izquierdas N²6, enero 2016, IDEA-USACH, ISSN 07185049, pp.169-191

\title{
Complejidades conceptuales sobre el colonialismo y lo postcolonial. Aproximaciones desde el caso del Pueblo Mapuche*
}

\author{
Conceptual complexities of colonialism and postcolonial. \\ Approaches from the case of the Mapuche people
}

\author{
José Luis Cabrera Llancaqueo*
}

\begin{abstract}
Resumen: Las independencias de las colonias europeas producidas en el siglo XX, abrieron una escena postcolonial en la que se han desarrollado distintos cuestionamientos a la persistencia de elementos propios del régimen colonial con posteridad a su desaparición, cuestionamientos que han dado lugar a corrientes de pensamiento críticas y programas políticos que buscan revertir dicha persistencia. En esta escena postcolonial los pueblos indígenas logran posicionarse de forma protagónica, de forma tardía si se considera su larga data de pueblo colonizado, a partir de la década de 1980. Sobre las razones históricas de este posicionamiento tardío, en particular de la situación colonial que vive el Pueblo Mapuche trata el siguiente artículo. Cuándo se inicia su condición colonial, de qué forma se ha reconfigurado a través del tiempo, la manera en que se ha analizado la crítica a la escena postcolonial y cuáles son los proyectos de salida al colonialismo son las preguntas que organizan este trabajo.
\end{abstract}

Palabras clave: Colonialismo, escena postcolonial, pueblos indígenas, Pueblo Mapuche.

\begin{abstract}
The independence of the European colonies produced in the twentieth century, opened a postcolonial scene in which we have developed different challenges to the persistence of elements of colonial rule with posterity to his disappearance, questions that have led to current and critical thinking political programs that seek to reverse such persistence. In this scene postcolonial indigenous peoples manage to position protagonist so, belatedly considering its longstanding colonized people, from
\end{abstract}

*Este artículo forma parte del la tesis doctoral "Pueblo Mapuche y conflictos etnopolíticos-territoriales en Chile y Argentina" realizada por el autor.

"Chileno, Doctor () en Procesos sociales y políticos en América Latina, Universidad de las Artes y Ciencias Sociales, Santiago, Chile. Miembro Grupo de Trabajo Kuifike. Docente Escuela Latinoamericana de Postgrados Universidad Artes y Ciencias Sociales. Correo electrónico: correojlcabrera@gmail.com 
the 1980s. On the historical reasons for this late position, especially the colonial situation the Mapuche people live in the following article. When the colonial condition starts, how it has been reconfigured over time, how has analyzed the criticism of postcolonial scene and what projects are out to colonialism are the questions that organize this work.

Keywords: Colonialisim, postcolonial scene, indigenous people, Mapuche people.

\section{Introducción}

Desde la década de 1980 los pueblos indígenas en América Latina se han posicionado como actores sociales y políticos autónomos en las sociedades nacionales en las que, producto de procesos históricos de conquista, despojo y asimilación, han debido desenvolverse ${ }^{1}$ (Canales Tapia y Rea Campos 2013). Una de las temáticas más recurrentes a partir de esta situación ha sido el colonialismo, cuestión que en aquellas fechas, a nivel global, se circunscribía a los casos de las colonias europeas que seguían manteniéndose con posterioridad a la Segunda Guerra Mundial, mientras que en el continente latinoamericano el colonialismo se pensaba como una realidad pasada, que tuvo su término con los movimientos independentistas de inicios del siglo XIX que pusieron fin al dominio español. Por tanto, el posicionamiento de los pueblos indígenas con un discurso crítico del colonialismo generó, y sigue generando, la necesidad de una nueva mirada sobre cuál ha sido el papel que estos pueblos tuvieron y tienen en los países del continente. No obstante lo anterior, la construcción de esta nueva mirada no ha resultado fácil ni exenta de problemáticas. En primer lugar, porque existen intereses y visiones políticas, económicas y académicas dominantes que se resisten a la modificación del relato histórico nacional que les ha permitido mantener dicha posición en la sociedad; y en segundo lugar, por una diversidad de lecturas, incluso contrapuestas, sobre cómo entender el colonialismo y cómo elaborar propuestas descolonizadoras, lo que deviene en una complejidad conceptual. Esta es la importancia que asume este artículo en la tarea de esclarecer a qué se hace referencia cuando se recurre al concepto colonialismo desde distintas interpretaciones.

En este sentido, el las siguientes líneas se busca discutir en torno a los principales tópicos sobre la temática colonialista e identificar cómo éstos se encuentran presentes en la realidad de los pueblos indígenas a partir del caso del Pueblo Mapuche. Es así como en el primer apartado se analizará a los principales autores que han asumido el estudio de la temática en

\footnotetext{
${ }^{1}$ Pedro Canales Tapia y Carmen Rea Campos, Claro de luz. Descolonización e "intelectualidades indígenas" en AbyaYala, siglos XX-XXI, Santiago de Chile, IDEA-USACH, 2013.
} 
las ciencias sociales, identificando sus definiciones y propuestas teóricas. Por lo tanto, ¿cuáles han sido los elementos sobre los que se han elaborado conceptualizaciones como colonialismo, escena postcolonial o régimen colonial? ¿A partir de qué contextos sociohistóricos y programas -tanto político como intelectuales- han surgido estas conceptualizaciones? Serán as interrogantes que guiaran el desarrollo de este apartado, con el objetivo de fijar las coordenadas teóricas sobre las que se analizará el caso mapuche.

El segundo apartado está dividido en dos partes. En primer lugar, se presenta una revisión histórica sobre cómo ha operado el colonialismo chileno sobre el Pueblo Mapuche, tanto en su versión hispana como republicana. Se profundizará, a partir de la revisión de fuentes secundarias proporcionadas por distintos exponentes, principalmente mapuche, las reconfiguraciones del colonialismo que ha experimentado este pueblo a lo largo de su Historia y sus manifestaciones en su realidad social. La segunda parte se aboca a analizar uno de los casos más emblemáticos y cercanos en el tiempo donde se pueden apreciar elementos colonialistas en la relación chileno-mapuche: la construcción de la central hidroeléctrica Ralco en Alto Bíobío. En este sentido, las preguntas que busca despejar este apartado son ¿cuáles han sido los mecanismos de reconfiguración del colonialismo que ha experimentado el Pueblo Mapuche?, ¿qué efectos han provocado en su organización social y en sus relaciones internas, así como también en su relación con el Estado y la sociedad chilena?

El tercer apartado, ofrece una problematización en cuanto a las salidas que se han teorizado para eliminar el colonialismo como relación social, poniendo especial énfasis en las estrategias políticas de "colonizar al colonizador" y los polémicos esencialismos estratégicos. Considerando esto último, se plantean escenarios de descolonización para el Pueblo Mapuche a partir de las expectativas que abrió el escenario político de cambio constitucional en Chile. La pregunta que organiza este apartado es ¿cuáles son las estrategias más viables para conseguir el objetivo en este nuevo contexto, considerando la trayectoria histórica que ha tenido el colonialismo sobre el Pueblo Mapuche y sus reconfiguraciones? A continuación de este tercer apartado, se presentan las principales ideas que se pueden concluir de este artículo, indicando las proyecciones investigativas que ofrece.

\section{Consideraciones iniciales respecto a la escena postcolonial y el colonialismo}

Las escenas postcoloniales que se abrieron con el fin de la Segunda Guerra Mundial, devinieron en la fuente de numerosos desafíos para las ciencias sociales, las cuales se habían constituido sobre las bases del discurso y el proyecto moderno. Éstas experimentaron un agotamiento producto de la crítica a los valores de conocimiento que sustentaban a dicho proyecto, tales como la universalidad del saber, la objetividad del 
José Luis Cabrera, Complejidades conceptuales sobre el colonialismo y lo postcolonial. Aproximaciones desde el caso del Pueblo Mapuche / Conceptual complexities of colonialism and postcolonial. Approaches from the case of the Mapuche people, Revista Izquierdas $N^{\circ} 26$, enero 2016, IDEA-USACH, ISSN 07185049, pp.169-191

conocimiento científico, la búsqueda de la verdad o la neutralidad de las técnicas de investigación que depositaban una fe ciega en la razón. En estas escenas postcoloniales, quizás la mayor crítica que sufrió la ciencia social fue su eurocentrismo y la constitución de una "geopolítica del conocimiento" que ubica jerárquicamente a Europa y la sociedad occidental por sobre otros espacios del mundo como África, Asia o América en lo que a producción de teorías y paradigmas sociales se refiere ${ }^{2}$. Esta crítica dejó en evidencia el ethos colonialista de las ciencias sociales modernas.

Numerosas fueron las teorías y programas de estudio postcoloniales que apostaban por procesos que revirtieran el colonialismo persistente en las sociedades de los países excolonias europeas que consiguieron sus independencias promediando el siglo XX, siendo ejemplos emblemáticos los casos de la India que consiguió su independencia de Gran Bretaña y Argelia que hizo lo propio respecto de Francia.

Dentro de los nombres más reconocidos de estas escenas postcoloniales se encuentran Frantz Fanon ${ }^{3}$, Edward Said ${ }^{4}$, Gayatri Spivak ${ }^{5}$, Aníbal Quijano; ${ }^{6}$ cada uno de ellos con sus

\footnotetext{
${ }^{2}$ Miguel Valderrama, Epistemología de las ciencias sociales, Santiago de Chile, ARCIS-SELA, 2002, 20; Walter Mignolo, Capitalismo y geopolítica del conocimiento. El eurocentrismo y la filosofía de la liberación en el debate intelectual contemporáneo. Buenos Aires, Ediciones del Signo, 2001.

${ }^{3}$ Frantz Fanon es considerados uno de los precursores del estudio de las escenas postcoloniales, identificando al colonialismo como la imposición de la soberanía extranjera en territorios conquistados y sometiendo a la población originaria, generando a su vez otras problemáticas vinculadas a la discriminación racial. Una de las conceptualizaciones que más destacan en la elaboración de Fanon para la comprensión y análisis de estas problemáticas es lo que Héctor Nahuelpan denomina colonialismo internalizado, es decir, la representación de sujetos como raza inferior o minorizados que transforma a la violencia y el tutelaje como las principales formas de integración-exclusión y de gobierno. Ver en Héctor Nahuelpan, "Las 'zonas grises' de las historias mapuche. Colonialismo internalizado, marginalidad y políticas de la memoria". Revista de Historia Social y de las Mentalidades, Numero1, Volumen 17, Santiago de Chile, 2013, 13. Para profundizar en el pensamiento de Fanon, ver Enrique Antileo, "Frantz Fanon wallmapupüle. Apuntes sobre el colonialismo y posibilidades para repensar la nación en el caso mapuche", Oliva Elena, Lucía Stecher, y Claudia Zapata (Eds.), Frantz Fanon desde América Latina. Lecturas contemporáneas de un pensador del siglo XX, Buenos Aires, Corregidor, 2012125 - 154; Frantz Fanon, Los condenados de la tierra, México, Fondo Cultura Económica, 2003 y Frantz Fanon Piel negra, máscaras blancas, Buenos Aires, Editorial Abraxas, 1973.

${ }^{4}$ Said fue un intelectual y activista político palestino, considerado uno de los más influyentes pensadores de las escenas postocoloniales, principalmente por su obra Orientalismo publicada originalmente en 1978, donde realiza una aguada crítica a los prejuicios que en occidente se construyen sobre la población oriental. Ver Edward Said, Orientalismo, Barcelona, Editorial Debate, 2002.

${ }^{5}$ Spivak es una pensadora India, que junto a otros estudiosos como Dipesh Chacravarty, forman parte de una corriente de pensamiento denominada Estudios Subalterno, cuya influencia ha llegado a América Latina. Su línea argumental se basa en la exclusión de la que son objeto los sujetos subalternos en la ciencia, filosofía e historiografía europea pretendida como universal. Ver Gayatri Spivak, Crítica a la razón postcolonial. Hacia una historia del presente evanescente. Madrid, Ediciones Akal, 2010 y Gayatri Spivak, "Estudios de la subalternidad: deconstruyendo Ia historiografía", Silvia Rivera Cusicanqui y Rossana Barragán (Eds.),
} 
particularidades dadas por sus contextos espacio/temporales y sus posiciones epistemológicas. Debido a esta variedad el contenido del concepto colonialismo y sus derivados como colonial, colonialidad, colonizador, colonos y colonizados tiende a superponerse y a complejizar la comprensión para aquellos que inician la lectura y el estudio de estos trabajos. Y esta complejidad se acentúa cuando entran a la escena de discusión, muy tardíamente considerando su realidad de colonizados, los pueblos indígenas a fines de la década de 1970. ¿Qué es el colonialismo y cómo se expresa en una realidad histórica concreta?, es una de las cuestiones a resolver para pensar en un proyecto de descolonización.

Jean Paul Sartre, intelectual francés que "demuestra temprana adhesión a la causa de Frantz Fanon... pese a que la academia francesa aún no ha asumido de manera explícita su pasado colonial", , señala en su ensayo Situaciones $V$ : Colonialismo y neocolonialismo que el colonialismo tiene un carácter sistémico aplicable en distintos contextos y contiene en su interior elementos económicos, sociales y psicológicos; amalgamados en un sistema político que conserva la soberanía extranjera en un territorio conquistado. Refiriéndose al caso de la colonización francesa en Argelia, Sartre sostiene que

La colonización no es un conjunto de azares, ni el resultado estadístico de miles de empresas individuales. Es un sistema puesto en ejecución hacia mediados del siglo XIX, que comenzó a dar frutos hacia 1880, entró en decadencia después de la Primera Guerra Mundial y en la actualidad se vuelve contra la nación colonizadora",

Pese a que podemos homologar las características que Sartre le atribuye al colonialismo como sistema a la condición de pueblos colonizados que tienen pueblos indígenas en América Latina, no podemos hacer lo mismo respecto a su periodificación. Esto se debe a la temprana colonización de los espacios territoriales americanos por parte de los españoles y los procesos históricos, sociales y políticos desarrollados por las elites criollas, lo que

Debates Post Coloniales: Una introducción a los Estudios de la Subaltenidad, La Paz, SEPHIS- Editorial Historias, 1997, 247-278.

${ }^{6}$ Quijano es considerado uno de los fundadores del llamado pensamiento decolonial, que se ha elaborado desde América Latina con una fuerte crítica al eurocentrismo. Su principal reconocimiento es la idea de la colonialidad del poder, donde persistente una colonialidad en las relaciones sociales (genero, étnicas, clase), relacionadas temporalmente con la empresa colonizadora europea. Ver Aníbal Quijano, "Colonialidad del poder. Cultura y conocimiento en América Latina”, Mignolo (ed), op. cit. 117-131 y Anibal Quijano, "Colonialidad del poder y clasificación Social", Santiago Castro-Gómez y Ramón Grosfoguel (Comps.), Giro decolonial, Reflexiones para una diversidad epistémica más allá del capitalismo global. Bogotá, Siglo del Hombre Editores, 2007, 93 - 126.

${ }^{7}$ Alison Spedding, Descolonización. Crítica y problematización a partir del contexto boliviano, La paz, ISEAT, 2011, 49.

${ }^{8}$ Jean Paul Sartre, Colonialismo y neocolonialismo. Situaciones V. Buenos Aires, Editorial Losada, $1965,21$. 
redundó en una tardía aparición de movimientos independentistas con carácter de liberación nacional al interior de los pueblos indígenas, lo que difiere del caso argelino. Esto permite identificar una primera características del colonialismo, la cual consistente en que éste adquiere una configuración específica dependiendo del espacio geográfico en que se ejecute; y a su vez experimenta reconfiguraciones producto de las trasformaciones políticas que experimenten las sociedades colonizadoras y colonizadas.

Haciendo un balance de lo expuesto hasta aquí, el colonialismo lo podemos interpretar como el resultado de una empresa de conquista, cuyos efectos se pueden apreciar, por un lado, en la estructura política fijada para establecer el estatus jurídico de colonizadores y colonizados (entiéndase el régimen colonial político, jurídico, estatal), lo que podemos llamar colonialismo formal.

Por otro lado, el colonialismo lo podemos interpretar como una relación social, donde se constata una asimetría dominador/dominado, en las que el colonizador se constituye como superior al colonizado. En el caso de los pueblos indígenas, la diferencia colonizadora original se constituyó en torno a las prácticas comunitarias de producción y reproducción de la vida, las que no concordaban con el ideal de progreso europeo. Con posterioridad, esta incongruencia devino en diferencias basadas en aspectos étnicos como la raza, los rasgos físicos o las prácticas culturales que dan paso a la discriminación y estereotipos de inferioridad.

Existe una tercera interpretación que pone en el centro del análisis el accionar de los colonos como agentes del colonialismo, provocando un giro en los enfoques más comunes que le otorgan centralidad a la instalación de un régimen colonial para definir el colonialismo. Esta novedosa interpretación es la que ha permitido vincular estrechamente la idea de colonialismo con la crítica al anti-eurocentrismo, en la medida que esta crítica se focaliza en exceso en los regímenes que las potencias europeas instalaron en los espacios geográficos invadidos y sus consecuencias. Un ejemplo de esto último es la siguiente afirmación:

(...) no obstante la gran variedad de temas, posturas teóricas y grupos de estudiosos y estudiosas en diferentes países que han participado en este campo académico, el principio fundamental de la perspectiva poscolonial es simple y claro: para comprender todas y cualquiera de las sociedades o grupos sociales existentes en el mundo de hoy, es imprescindible tener en cuenta los procesos del colonialismo europeo, es decir, los procesos a través de los cuales los países de Europa occidental, y luego otros países gobernados por descendientes europeos identificados 
con su cultura, llegaron a controlar la vasta mayoría de la superficie terrestre a principios del siglo $\mathrm{XX}^{9}$.

Al hacer este giro y poner el énfasis en el accionar de los colonos, la interpretación del colonialismo deja de tener un componente esencialmente europeo, ampliando el concepto. Aquí se habla de un "colonialismo de colonos" que opera sobre la base de dinámicas sociales que establece el colono a su llegada, sin obedecer a una planificación política llevada a cabo por un Estado extranjero que busca extender su dominación exportando sus valores y modelos político-sociales. De esta forma entra dentro del concepto de colonialismo la dominación ejercida por colonos, sin que necesariamente provengan o desciendan de Europa, lo que genera una crítica no solo al eurocentrismo, sino también al anti-eurocentrismo, aquel que en su crítica a Europa no puede desprenderse de ella. Esta interpretación de "colonialismo de colonos" es utilizada por el historiador surcoreano Jo SungHoonn, que a partir de ejercicios comparativos sobre el colonialismo en Asia en general, y del colonialismo japonés en Corea en particular, afirma que el colonialismo es un fenómeno global que no se circunscribe a la acción de las potencias europeas, lo que le ha permitido establecer paralelos con el caso mapuche que le permitirían sustentar su afirmación, como se examinará más adelante.

Así como la interpretación del "colonialismo de colonos" viene a cuestionar aspectos que parecían consolidados como verdades inmodificables en los estudios sobre colonialismo y que invisibilizaban colonialismos no-europeos, las corrientes intelectuales postcoloniales no han estado exentas de críticas. Por ejemplo, Grínor Rojo, Alicia Salomone y Claudia Zapata afirman que:

Si, por un lado, los críticos postcoloniales son teóricos sobre cuyas ideas gravitan los axiomas más bien incoloros del postestructuralismo y/o el posmodernismo, por otro, ellos quieren pensar su trabajo como si éste estuviera dando forma a un dialogo de continuidad consecuente con el pensamiento anticolonial y antineocolonial de las décadas del cincuenta sesenta y setenta. Nosotros estimamos que hay ahí la pretensión de una síntesis que los datos que disponemos no autorizan, que los proyectos intelectuales de Frantz Fanon y el Ché Guevara en una esquina, y Gayatri Spivak y Homi Bhabha en la otra, no sólo no vertebran un continuum lógico genuino, sino que ni siquiera resultan compatibles ${ }^{11}$.

\footnotetext{
${ }^{9}$ Spedding, op. cit., 41

${ }^{10}$ Jo SungHoon, 2014. Presentación en la $\mathrm{I}^{\circ}$ Jornadas Internacionales Intelectualidades Emergentes, Santiago de Chile: IDEA-USACH, 2014.

${ }^{11}$ Grínor Rojo, Alicia Salomone y Zapata, Claudia, "Postcolonialidad y nación: algunos aspectos de ladiscusión teórica", Alejandra Castillo et. al (Eds.), Nación, Estado y Cultura en América Latina, Santiago de Chile: Ediciones Facultad de Humanidades Universidad Chile, 2003, 18.
} 
Asimismo, poniéndose en una perspectiva crítica indígena en contra del colonialismo, Spedding plantea que "en el curso de los años 1990, la causa de los pueblos indígenas fue asumida por las redes de activistas de izquierda internacional, se creó el contexto para pasar de la perspectiva analítica del poscolonialismo a la propuesta política de la descolonización" ${ }^{\text {"12 }}$, deslizando un cuestionamiento a ciertas estudiosas y los estudiosos de las escenas postcoloniales, en tanto que su trabajo no salía de las academias europeas y no se consolidaba en un proyecto político. Sin embargo, al establecer que son las redes de izquierda internacional las que crean el contexto para un proyecto político descolonizador, desconoce y disminuye la incidencia política de los pueblos indígenas en su consolidación como actores relevantes en la década de 1990. Esto juicio también se puede hacer extensivo al pensamiento decolonial latinoamericano ${ }^{13}$, pues según G. de Mussyy Valderrama éste "es un proyecto de desprendimiento epistémico de raíz política y social, mientras que la crítica postcolonial (...) es un proyecto de transformación que opera (...) en la academia francesa y estadounidense", 14 , sin embargo,

Si bien es cierto que Quijano o Mignolo dan una importancia a los indígenas dentro de su programa, no es posible advertir una liberación de estos en particular; sino, más bien, de todos los sectores que han vivido los efectos nocivos de la colonialidad eurocéntrica y capitalista en América Latina. A esto se suma que los impulsores del cambio epistémico (Quijano, Mignolo, Dussel, Lander Walsh etc.) no son indígenas, por lo que el pensamiento decolonial fácilmente puede ser considerado como una instancia de dominación e indigenismo; es decir, un programa donde los no indígenas pretenden hablar por ellos y fijar su agenda política ${ }^{15}$.

Como se puede ver, el colonialismo es una realidad concreta que se constata en las sociedades que vivieron una experiencia de invasión e imposición de la soberanía extranjera sobre la población originaria, la cual da origen a una situación colonial donde se aprecian relaciones asimétricas dominador/dominado. A partir de esto último se constituyen escenas críticas postcoloniales que se expresan en debates intelectuales y proyectos políticos que no son homogéneos, llegando incluso a disputas entre distintos discursos críticos. En lo que viene a continuación, se analizará la escena particular de colonialismo y la discusión político-intelectual que ha protagonizado el Pueblo Mapuche en el contexto chileno.

\footnotetext{
${ }^{12}$ Spedding, op. cit., 38

${ }^{13}$ Para profundizar sobre el pensamiento decolonial latinoamericano, ver nota $\mathrm{N}^{\circ} 6$.

${ }^{14}$ Luis G. de Mussy y Miguel Valderrama, Historiografía postmoderna. Conceptos, figuras, manifiestos, Santiago de Chile, Ril Editores-Universidad Finis Terrae, 2010, 86.

${ }^{15}$ José Luis Cabrera Llancaqueo y Augusto Aillapan Paillafil, Machi mongen tani Santiago warria mew. Vida de un machi en la ciudad de Santiago, Santiago de Chile, Grupo de Trabajo Kuifike, 2013, 46.
} 


\section{Colonialismo sobre el Pueblo Mapuche}

El colonialismo experimentado por el Pueblo Mapuche se aparta de la trayectoria histórica vivida por otros pueblos indígenas del continente. Esto se debe, principalmente, al proyecto de conquista que se desplegó sobre él. Los españoles emprendieron su empresa de conquista al sur del Perú instalando un régimen colonial formal, tal como lo habían hecho en el resto de los territorios. Sin embargo, la llamada Araucanía fue uno de los territorios que nunca lograron conquistar completamente; de ahí que al momento de su independencia, Chile fuera aún una capitanía general con mandos militares a cargo de las instituciones políticas ${ }^{16}$. Esto permite comprender que en las esferas políticas hispanas, y pese a los parlamentos celebrados, la percepción era que la conquista iniciada en el siglo XVI no estaba completa; no obstante a que los actores que convivían en la frontera, con el pasar de los años llevaron a cabo relaciones que en la práctica significaban el cumplimiento de lo establecido en los parlamentos (la frontera), y a su vez el reconocimiento del territorio autónomo y la soberanía mapuche.

Todos estos elementos llevan a sostener que el colonialismo llevado a cabo por el Estado chileno desde la década de 1880 del siglo XIX no sólo son relaciones asimétricas entre chilenos y mapuche, sino que lo emprendido por la República de Chile sobre lo que sus elite políticas denominaban Araucanía representa un continuum del proyecto colonizador con elementos propios del período de expansión que va desde el siglo XV al XVIII. Esta idea se puede considerar una reinterpretación del colonialismo que ha experimentado el Pueblo Mapuche en su relación con Chile, puesto que se diferencia de la lectura que ha predominado en historiadores mapuche que asumen una perspectiva descolonizadora y consideran que la situación colonial se inaugura sólo a partir del siglo XIX, como cuando Sergio Caniuqueo afirma que "contemplar el siglo XX, para nosotros los mapuche, implica retomar nuestra historia, que actualmente se encuentra suspendida, al igual que nuestra soberanía" 17 .

Ahora bien, como los procesos históricos no se repiten de forma idéntica por la influencia de los contextos temporales, es necesario establecer las diferencias con la forma clásica que había adoptado la colonización hispánica en los territorios conquistados, ya que la instalación de un régimen colonial formal, con instituciones políticas que lo validaran y lo eximiera de críticas, no podía llevarse a cabo en el siglo XIX. Cabe recordar que las conquistas hispánicas y el régimen colonial aplicado a los indígenas no eran objeto de una crítica en sí mismos como ocurre a partir del siglo XX, sino de una crítica ético-valórica a

\footnotetext{
${ }^{16}$ Osvaldo Silva Galdames, Historia de Chile 2. La colonia 1601-1800, Santiago de Chile, Copesa, 2005.

${ }^{17}$ Sergio Caniuqueo, "Siglo XX en Gulumapu: de la fragmentación del Wallmapu a la unidad nacional mapuche. 1880-1978", Pablo Mariman et al. Escucha wainka. Cuatro ensayos de historia nacional mapuche y un epílogo sobre el futuro", Santiago de Chile, Lom Ediciones, 2006, 129.
} 
la forma a cómo se estaba llevando a cabo la colonización, principalmente en lo que se relacionaba con el trato que recibía el indígena. Esta crítica fue llevada a cabo, principalmente, por las órdenes religiosas en contra de la institución de la Encomienda, destacándose en el caso mapuche la función del jesuita Luis de Valdivia, quien promovió la estrategia de "guerra defensiva" en contra de los araucanos, reemplazando las campañas militares por misiones. Pese a que la guerra defensiva fue desechada por la presión de los encomenderos, la crítica de las órdenes religiosas puede interpretarse como una de las formas de contrainsurgencia planteadas por Ranajit Guha, aquella donde, en apariencias, el colonizador sintoniza con los intereses de los colonizados, pero que no busca cambiar su estatus, puesto que sigue manteniendo su soberanía en la colonia ${ }^{18}$. En definitiva, lo que se busca es que el régimen colonial funcione bien y no haya espació para una insurgencia liberalizadora.

Pero quizás la diferencia principal sea que el fundamento de la empresa conquistadora chilena no tiene sustento ideológico en la evangelización de los indios para que encontraran la salvación, sino que pretendía integrarlos a la civilización ilustrada promovida por el liberalismo positivista del siglo XIX, lo cual era incompatible con la existencia del mapuche como tal. Aquí aparece una característica del colonialismo sobre el Pueblo Mapuche en el siglo XIX, la cual consiste en buscar su asimilación a la nación chilena bajo el supuesto de que la ciudadanía haría un "Chile para los chilenos, aunque, en rigor, no lo era para todos por igual" ${ }^{\prime 19}$. De esa promesa incumplida de igualdad para todos, se desplegará el colonialismo sobre la sociedad mapuche, estableciendo la inferioridad de sus miembros frente a los chilenos.

Una última diferencia que podemos destacar entre la colonización hispánica y la chilena, se constata en la promoción de la colonización extranjera por parte del Estado de Chile en el territorio mapuche, lo cual puede dar espacio para la interpretación de colonialismo de colonos que plantea en su trabajo Jo SungHoon, pese a que si existió una planificación estatal. La presencia de este tercer actor ha sido fuente de numerosos conflictos territoriales cuyos orígenes se encuentran en el colonialismo mismo, transformándolos en un ejemplo de conflictos políticos de difícil resolución. Las principales promociones de colonización post reducción fue la colonización alemana en la zona de Llanquihue, coordinada por Vicente Pérez Rosales, y la suiza en Malleco.

\footnotetext{
${ }^{18}$ Ranajit Guha, "La prosa de contra insurgencia", Debates Post Coloniales. Una introducción a los Estudios de la Subaltenidad. Silvia Rivera Cusicanqui y Rossana Barragán (Eds), op. cit.33 - 72.

${ }^{19}$ Tito Tricot, Autonomía. El Movimiento Mapuche de Resistencia, Santiago de Chile, Ceibo Ediciones, 2012,88 .
} 
Respecto a este último caso, Sergio Caniuqueo expone los planteamientos de Alberto Dufey Castro sobre la colonización suiza, donde señala que, en un principio, mapuche y colonos suizos mantenían buenas relaciones, agregando lo siguiente:

(...) en un momento existió la posibilidad de generar una alianza con el colono, si éste no se hubiera aprovechado del patrimonio mapuche para expandirse económicamente. Dufey menciona, basado en cartas emitidas por colonos suizos a sus familiares en Europa, que los mapuches eran flojos y desconfiados, aunque en las mismas cartas hablaban de la colaboración que realizaban con ellos, mencionando las amistades entre los niños. El autor planteó que la relación de colaboración se vio eclipsada con los años debido a la acumulación económica y el prestigio de los suizos, estableciéndose una relación de dominador y dominado en que el mapuche pasa a ser el trabajador agrícola y su mujer la sirvienta en la casa de los colonos ${ }^{20}$.

Aquí podemos apreciar el carácter sistémico del colonialismo señalado por Sartre, puesto que las principales diferencias son las económicas, las sociales y las psicológicas, y en ningún caso se derivan de lo racial, sino de la voluntad política de imponer la soberanía chilena y eliminar la mapuche.

\section{Reconfiguraciones históricas del colonialismo chileno}

Como se mencionó anteriormente, el colonialismo tiene la capacidad de reconfigurarse a partir de las transformaciones experimentadas por las sociedades, tanto colonizadoras como colonizadas. El triunfo militar chileno significó la desestructuración de la sociedad mapuche y la desposesión de su territorio:

(...)al referirnos a la formación del Estado vinculada a continuos procesos de despojo debemos remitirnos tanto a la acumulación de poder político, económico y/o simbólico de orden colonial, derivados de un acto de conquista y de prácticas de desposesión territorial o material que lo hicieron o hacen posible, como asimismo a un modo particular de construcción sociopolítica y cultural que ha propiciado la desposesión, colonización de cuerpos y subjetividades Mapuche, bajo disciplinas laborales, religiosas y escolares a las cuales se les atribuye un carácter civilizatorio. Este proceso colonizador ha permitido incluso la internalización de complejos de inferioridad en distintas generaciones Mapuche. Esto último constituye una de las dimensiones más potentes y desgarradoras del fenómeno colonial $^{21}$.

\footnotetext{
${ }^{20}$ Sergio Caniuqueo, op cit., 155.

${ }^{21}$ Héctor Nahuelpan, "Formación colonial del Estado y desposesión en Ngulumapu", Héctor Nahuelpan, et. al., Ta iñ fijke xipa rakizuameluwün. Historia, colonialismo y resistencia desde el país Mapuche. Temuco, Ediciones Comunidad de Historia Mapuche 2012, 122.
} 
De lo señalado por Nahuelpan se desprende que dentro del proceso colonial, el despojo territorial tiene vital importancia. Así es como en paralelo a la desposesión se desarrolló el proceso reduccional, consistente en la entrega de Títulos de Merced correspondientes a pequeños espacios denominados reducciones indígenas, las que Pedro Cayuqueo cataloga como verdaderos "campos de concentración",22. En su nueva condición -impuesta- de chilenos, los mapuche debieron acomodar sus formas de vida y organización social incorporando la idea propiamente moderna del progreso y establecer relaciones mercantilcapitalistas entre ellos y con sus vecinos chilenos, suizos y alemanes, conformándose la relación asimétrica colonialista que, según Sergio Caniuqueo, se dio por "reclutamiento social", es decir
(...) por elementos que dan forma (...) a estructuras que necesitan múltiples procesos, los cuales se articulan como sistemas, cuyas características son: la retroalimentación, la capacidad de modificarse ante los estímulos de la sociedad o de los grupos humanos, articulación con otros sistemas y flujos estimulados por elementos subjetivos, objetivos y objetivables, cuyas funciones implican una manera de establecer una coherencia social de los $\operatorname{grupos}^{23}$.

Muchos son los procesos que han actuado y reconfigurado el colonialismo chileno que se ha desplegado sobre el Pueblo Mapuche, comenzando por la ya mencionada incorporación de relaciones mercantilistas con los colonos; la posterior aparición de un mercado de tierras y la entrada del sistema financiero; el proyecto industrial desarrollista de la primera mitad del siglo XX; el proceso de reforma agraria; y la transformación neoliberal del Estado y la Sociedad chilena en fechas más cercanas.

Todos estos procesos también actúan y dejan su efecto en la sociedad mapuche. Destacamos aquí, en primer lugar, los que se relacionan con la organización política mapuche que a principios del siglo XX comienzan a desplegarse dentro de los marcos estatales de representación sin renunciar a su identidad mapuche, hasta las organizaciones autónomas de sistema político chileno que emergen desde la década de 1980. En segundo lugar, la migración hacia los centros urbanos en busca de nuevas y mejores perspectivas, las que se van a modificar en vista de que las aspiración del mapuche migrante aumenta y su destino sean los estudios superiores, lo que también se entrelaza con el giro experimentado por las organización política, provocando disputas y deslindes generacionales que dan cuerpo a nuevas generaciones políticas. Por último, en tercer lugar, el impacto de las transformaciones neoliberales, que en el caso mapuche se concretizó con el Decreto Ley

\footnotetext{
${ }^{22}$ Pedro Cayuqueo, Solo por ser indios y otras crónicas mapuches. Santiago de Chile, Catalonia, 2012.

${ }^{23}$ Caniuqueo, op. cit., 135
} 
2.568 que dividió la propiedad comunitaria indígena y activó un nuevo proceso de creación de mercados de tierra orientados a la industria forestal.

A esta sintetizada caracterización de las reconfiguraciones del colonialismo chileno sobre el Pueblo Mapuche, no podemos dejar pasar el aspecto demográfico, puesto que la migración hacia centros urbanos ha provocado que más del $60 \%$ de la población mapuche se encuentre fuera del Wallmapu ${ }^{24}$ histórico, generando formas de colonialismo vinculadas a la discriminación racial que propicia la sociedad mayoritaria sobre un otro que es construido como inferior. En este sentido, Enrique Antileo en sus estudios sobre la población mapuche migrante o "diáspora mapuche", va a poner énfasis en el rol que cumple el aspecto racial en la clasificación y estratificación de la sociedad chilena en que deben desenvolverse los mapuche. Es así como identifica mecanismos de selección de rasgos, que se encuentran naturalizados y marcan a grupos de población, atribuyéndoles un destino en la jerarquía social y en las relaciones de producción, explicando el por qué existen trabajos específicos para mapuche en la ciudad:

En términos concretos, estas referencias me permiten discutir la noción de trabajo racializado para el caso de sirvientas y panaderos mapuches en un marco general donde la raza ha operado como mecanismo de clasificación en la estructura colonial, cuya consolidación no solo describe el pasado, sino se abre al análisis de continuidades y expresiones contemporáneas de racismo. La idea central es poder sostener la configuración histórica del trato racial hacia el otro indígena en Chile, condición que tiene diversas manifestaciones, pero que en el ámbito laboral puede observarse en el funcionamiento y vigencia de imaginarios sobre el "indio" y su lugar confinado en la estructura social. Es decir, en el caso de la población mapuche en la ciudad de Santiago, a través de una breve mirada a las estadísticas sociales y testimonios laborales, es posible hablar de las marcaciones que la sitúan como un rostro de la exclusión ${ }^{25}$.

Lo planteado por Antileo nos muestra un aspecto de continuidad colonialista en la construcción del otro mapuche en las ciudades chilenas, puesto que podemos encontrar las mismas marcaciones de carácter racial que Caniuqueo identificaba en la realidad de sirvienta a la que se vio forzada la mujer mapuche en las haciendas que los colonos establecieron en Wallmapu. Sin embargo, esta continuidad se desarrolla en un contexto social nuevo dado por el contexto urbano, una manifestación más de que el colonialismo se reconfigura históricamente.

\footnotetext{
${ }^{24}$ Denominación que los mapuche le dan a su territorio histórico, derivado del mapudungun.

${ }^{25}$ Enrique Antileo, "Trabajo racializado. Una reflexión a partir de datos de población indígena y testimonios de la migración y residencia mapuche en Santiago de Chile". Meridional. Revista chilena de estudios latinoamericanos, Número 4, Santiago de Chile, 2015, 90.
} 


\section{Ralco, ejemplo del colonialismo en su reconfiguración neoliberal}

A continuación presentaremos un caso paradigmático donde el colonialismo chileno queda demostrado en su aspecto institucional y social, pero sobre todo porque su esencia pasa por la imposición de la soberanía chilena en un territorio ocupado militarmente. Estamos hablando del conflicto generado por la construcción de la mega represa Ralco, llevada a cabo por la transnacional energética Endesa ${ }^{26}$, enmarcada en el proceso de "modernización compulsiva"27 desarrollado por el Estado chileno en la segunda mitad de la década de 1990. Este caso se reavivó en la memoria del Pueblo Mapuche debido al fallecimiento de una de sus protagonistas en 2013, Nicolasa Quiltreman, quien junto a su hermana Berta se transformaron en el símbolo de la resistencia en contra del proyecto energético que el gobierno de Eduardo Frei Ruiz-Tagle se empecinaba en llevar a cabo durante el histórico y significativo 1997, año bisagra en la movilización mapuche contemporánea ${ }^{28}$.

Muchas fueron las muestras de apoyo a las hermanas Quiltreman, tanto mapuche como nomapuche, y con ello el apoyo al pueblo mapuche-pewenche. Sin embargo, también fueron muchas las críticas que se dejaron caer sobre Berta y Nicolasa cuando el proyecto prosperó y recibieron la permuta correspondiente, en vista a que la represa inundaría sus tierras.

Pedro Cayuqueo señalaba en 2011 respecto a aquellos que mostraron solidaridad en su momento:

Eran fácilmente identificables allí a la sombra del emblemático Volcán Callaqui, en tierras de la Comunidad Quepuca Ralco; en su mayoría rubios, de apellidos vinosos, oriundos del barrio alto capitalino, ex alumnos de la Pontificia y del Verbo Divino, y fanáticos como pocos de los deportes extremos. Furibundos opositores a los planes de Endesa-España, criticaban por igual a la empresa como a las familias locales que aceptaban -casi siempre regañadientes- dar finalmente su consentimiento al polémico megaproyecto apadrinado por Frei.

Nunca supe qué les interesaba defender más; si las aguas del río Bíobío o los derechos territoriales y culturales de los mapuche-pewenche. Nunca supe que les molestaba más; si la violación flagrante de estos derechos perpetrada por la

\footnotetext{
${ }^{26}$ Jessabel Guamán Flores, "Pascua Lama y Ralco en la década de la emergencia indígena. Notas para una historia indígena comparada en Chile, 1990-2010”, Historias que viven. Revista de estudiantes de Historia, Número6, Santiago de Chile, 61- 83.

${ }^{27} \mathrm{El}$ concepto del "modernización compulsiva" es utilizado por Víctor Toledo para nombrar el conjunto de transformaciones llevadas a cabo en territorio Mapuche, dentro del marco de modernizaciones neoliberales desarrolladas en Chile a partir de 1975. Ver Víctor Toledo, Pueblo Mapuche, derechos colectivos y territorio: desafíos para la sustentabilidad democrática, Santiago de Chile, Programa Chile Sustentable, 2005, 18.

${ }^{28}$ Fernando Pairican, Malon. La rebelión del movimiento mapuche 1990-2013, Santiago de Chile, Pehuén Ediciones, 2014 y Tricot, op.cit.
} 
Concertación o despedirse y para siempre de sus excitantes bajadas en rafting. De haber llegado a expulsar a Endesa, seguro, habrían continuado con los pewenche, bastante poco dados estos últimos al ecologismo profundo o al conservacionismo. No lograron ni lo uno ni lo otro, por suerte. Apenas las cosas se pusieron negras (es decir, hizo su aparición la fuerza pública con su habitual delicadeza interétnica), abandonaron el barco ${ }^{29}$.

Lo descrito por Cayuqueo sólo viene a confirmar una crítica que se ha venido desarrollando en contra del ecologismo radical por parte de los pueblos indígenas, quienes reclaman ser utilizados por estos grupos para conseguir sus propios fines, sin esperar que ellos logren instalar su agenda política y alcanzar sus objetivos, al respecto Toledo señala:

Las transnacionales ecologistas, que durante algún tiempo fueron apoyo mutuo con los indígenas, aportando sus competencias de advocacy para incidir en las redes de policy-making globales, hoy no vacilan en dejar a un lado, o incluso atropellar, a sus difíciles, lentos y circunstanciales aliados indígenas. El pragmatismo de los conservacionistas, y su sentido de oportunidad y timing, les indica que ya no tienen tiempo que perder en sutilezas, en la urgencia de conservar la naturaleza y constatando que existen escasas ventanas de oportunidad en el actual proceso de redefinición de regímenes internacionales. Tienen muy en claro su agenda -lo suyo nunca fueron los derechos indígenas-, y que en las circunstancias globales no queda tiempo, y que 'así de serio es el asunto' 30 .

Esta expresión del colonialismo no sólo se ve en la utilización de los indígenas, sino que también en no pocos sectores de la sociedad chilena que establece automáticamente un vínculo entre pueblos indígenas y ecologismo, fomentando una visión esencialista de los mapuche que coincide más con las interpretaciones que niegan la existencia del Pueblo por no conservarse como en los tiempos pretéritos, como si el tiempo no dejara huellas.

Por otra parte, está la crítica al acto de aceptar el acuerdo con Endesa y recibir la compensación. No son pocas las voces que acusan a Berta y Nicolasa de "venderse al capitalismo" y "traicionar la causa". ¿Qué podían hacer las hermanas frente a la fuerza de los hechos (la del gobierno y Endesa)? Tampoco ha faltado el argumento de las tierras improductivas, que la cantidad de dinero no se compara con la que recibe un campesino chileno empobrecido por sus tierras más productivas. Cabría preguntarse ¿cuántas son las ganancias de Endesa cada vez que se abren las compuertas para dejar pasar cientos de metros cúbicos? ¿Son proporcionales esas cifras con las recibidas por Berta y Nicolasa?

\footnotetext{
${ }^{29}$ Pedro Cayuqeo, "Ecologistas de postal", en Mapuexpress, 14 de mayo de 2011, http://www.mapuexpress.net/content/publications/print.php?id=5046 (Visitado el 25 de mayo de 2015)

${ }^{30}$ Víctor Toledo, "Políticas Indígenas y Derechos Territoriales en América Latina: 1990-2004", Pablo Dávalos (Comp.), Pueblos indígenas y democracia en América latina, Buenos Aires, CLACSO, 2005, 74-75.
} 
Efectivamente estamos frente a una nueva forma de acumulación propia de los tiempos del neoliberalismo globalizado, lo que David Harvey a conceptualizado como "acumulación por desposesión", donde la productividad no es la fuente de la riqueza, sino las industrias intensivas de extracción de recursos naturales como el agua. No hay productividad, pero sí hay ganancia; y ni Nicolasa, ni Berta, ni ninguna familia pewenche vio esa ganancia producida con las aguas de su territorio ancestral. No se trata sólo de propiedad y dinero, sino de soberanía y derechos colectivos de un pueblo que fueron vulnerados ${ }^{31}$.

\section{Salidas para el colonialismo chileno}

Pensar en salidas al colonialismo desde la perspectiva del colonizado es una tarea compleja, puesto que constantemente se presentará la posibilidad de encontrarlas a través de la estrategia de colonizar al colonizador, tal como señala Sartre al afirmar que el sistema colonialista se volvía contra la nación colonizadora.

En un estudio sobre el colonialismo en Rapa Nui, Jorge Iván Vergara, Hans Gundermann y Rolf Foerster rescatan la elaboración del antropólogo Ernst Gellner, quien señala que

(...) la institución moral anticolonial puede ser formulada en términos de dos proposiciones: (1) la desigualdad entre colonizador y colonizado es moralmente intolerable; (2) por lo tanto, los colonizados deben ser descolonizados, se les debe conceder la independencia política, para que se vuelvan similares a los colonizadores -y lo más rápido posible. Los anticolonialistas no han distinguido entre estos dos puntos y han considerado generalmente que son sólo uno, o han pensado que el segundo se deriva obvia e inevitablemente del primero. No es así. Las dos ideas son totalmente independientes. Es posible afirmar la primera pero no estar en absoluto comprometida con la segunda [...] Es posible rechazar apasionadamente la desigualdad entre colonizado y colonizador y sin embargo no creer que la descolonización sea indispensable. La simetría moral -aunque no mucha gente lo haya pensado- puede garantizarse de manera igualmente efectiva por lo contrario: no por la descolonización sino por la colonización del colonizador ${ }^{32}$.

Colonizar al colonizador es invertir la relación colonialista sobre la base de los mismos mecanismos sistémicos utilizados por los colonizadores para constituirla como asimétrica, es decir, presentar a los colonizados como superiores. Esto lo podemos constatar en la mencionada relación entre el ecologismo y las luchas indígenas, donde los primeros pretenden encontrar en los indígenas la llave maestra que abrirá los caminos de salida a la

\footnotetext{
${ }^{31}$ Toledo, Pueblo Mapuche...op cit.

${ }^{32}$ Jorge Vergara, Hans Gundermann y Rolf Foertster, Estado, conflicto étnico y cultura. Estudios sobrepueblos indígenas en Chile. Antofagasta: Universidad Católica del Norte/QILLQA - Universidad de Antofagasta, 2013, 188.
} 
crisis medioambiental, en cuanto sus costumbres y prácticas socioculturales serían moralmente superiores que la de los formados en la sociedad occidental.

Esto ha sido denominado como esencialismo indígena, similar a los esencialismos por los que fueron criticados los llamados Estudios Subalternos al utilizar fundamentos esencialistas como la raza o la sangre, con el fin de que cumplan la función de cemento social que proporciona la igualdad entre los sujetos subalternos; es decir, utilizar los mismos fundamentos teóricos criticados por el programa subalterno. Haciendo una apología del programa, Spivak responde a los cuestionamientos señalando que

(...) existen elementos en su texto [el del Grupo de Descolonización] que justifican la lectura de su proyecto de recuperación de la conciencia de los subalternos como un intento de desmontar esta metalepsis historiográfica masiva y 'situar' al efecto del sujeto como subalterno. Habría que leerlo, entonces, como un uso estratégico del esencialismo positivista en aras de un interés político escrupulosamente visible ${ }^{33}$.

Alison Spedding, considerando la realidad de los pueblos indígenas en Bolivia, sugiere que esta defensa no es aplicable en el caso de estos pueblos en el país andino, lo que también es aplicable al caso del Pueblo Mapuche. Ella señala tener

(...) la impresión que las y los esencialistas bolivianos, aunque estarían persiguiendo estrategias políticas, asumen sus esencialismos ingenuamente y como descripciones de la realidad, y sin considerar que apuntan a conclusiones como que al fin, sólo alguien que es indígena puede realmente comprender la cultura indígena, y por lo tanto, la convivencia con no indígenas es imposible y sólo queda la segregación y, en el peor de los casos, la limpieza étnica ${ }^{34}$.

Consideración estas aprensiones, y en algunos casos sometiéndolas a discusión y a juicio crítico que cuestiona la supuesta incapacidad de los indígenas para diferenciar entre el uso estratégico de un discurso para producir unidad interna y otro uso que promueve purismos étnico raciales, se han realizado aportes desde las ciencias sociales para comprender el colonialismo chileno sobre el Pueblo Mapuche, no tan solo como parte de una escena de crítica postcolonial, sino como un proyecto autónomo que busca la descolonización. Es así como en historiografía es posible constatar, a partir de 1990, una producción realizada por investigadores mapuche que intenta reinterpretar la construcción de la historia del Pueblo Mapuche hecha por la historiografía nacional chilena, con un enfoque descolonizador. Es decir, sintonizando con sectores movilizados del Pueblo Mapuche que han incorporado en

\footnotetext{
${ }^{33}$ Spivak, "Estudios de la subalternidad...op. cit., 257-258.

${ }^{34}$ Spedding, op. cit. 100.
} 
su proyecto político la idea autonómica y libredeterminista ${ }^{35}$. En estas escrituras mapuche se pueden advertir distintas formas en que al mapuche, como actor político e histórico, se le ha invisibilizado desde distintas esferas políticas e ideológicas chilenas. Por ejemplo, Claudio Barrientos reflexionando sobre el trabajo de Sergio Caniuqueo, señala que éste:

(...) nos obliga a descentrar nuestras percepciones sobre izquierda y progresismo, así como también sobre conservadurismo y colaboracionismo con la dictadura. En un sugerente análisis documental (...) nos plantea que la experiencia histórica y política (...) no responde a parámetros de izquierda o derecha, que más bien son sujetos que desde su condición de subalternidad elaboran estrategias independientes y autónomas para impulsar proyectos propios en beneficio de sus comunidades ${ }^{36}$.

A partir de lo anterior, es posible identificar una crítica a cómo la matriz de interpretación del espectro político chileno, clasificado históricamente en izquierda, centro y derecha, es impuesta en la lectura del accionar político de los mapuche. Sin embargo, también es posible encontrar una autocrítica, como la realizada por Nahuelpan a la excesiva politicidad que han adquirido los análisis sobre los mapuche realizados por mapuche, centrándose en proyectos, organizaciones y líderes del movimiento, pero excluyendo a aquellos que más experimentan el colonialismo en su vida cotidiana: sirvientas, jardineros o panaderos, migrantes que se ven obligados a desarrollan estos trabajos de "indios" en una ciudad que les resulta ajena ${ }^{37}$.

Sin buscar imponer una agenda política al movimiento mapuche, es posible leer el actual momento en la relación entre el Estado y la sociedad chilena con el Pueblo Mapuche como una encrucijada en la que los esencialismos, por más que puedan ser estratégicos, no ofrecen una salida. En este sentido, una importante salida para la actual encrucijada, agudizada con la muerte del matrimonio Luchsinger-Macay y la condena al machi Celestino Cordoba en 2014, es la que plantean Pedro Cayuqueo y Fernando Pairacan:

Lo charlábamos hace poco con Fernando Pairican, un joven y destacado historiador mapuche. Destrabar el conflicto pasa por llevar la lucha territorial a un plano mucho más ciudadano, de conquista de derechos civiles, culturales y políticos, 'algo similar a lo obrado en Estados Unidos por los afroamericanos', me comentó. Vaya si tiene razón. La Araucanía es ante todo un caso de comunidad regional fallida. Enmendar ese error es responsabilidad del Estado pero también de la ciudadanía. ¿Qué se

\footnotetext{
${ }^{35}$ Cabrera Llancaqueo y Aillapan Paillafil, op cit., 37-38. Un análisis en profundidad sobre cómo la discusión postcolonial influye (principalmente la de Fanon) en el movimiento mapuche y sus pensadores, puede verse en Enrique Antileo, "Frantz Fanon...,op. cit.

${ }^{36}$ Claudio Barrientos, Aproximaciones a la cuestión mapuche en Chile. Una mirada desde la historia y las ciencias sociales, Santiago de Chile, Ril Editores, 2014,16.

${ }^{37}$ Nahuelpan, "Las 'zonas grises'..., op cit.
} 
requiere? Que la política ocupe el lugar que le corresponde. Y la existencia de puentes interculturales que posibiliten el diálogo ${ }^{38}$.

Plantear esta salida al conflicto -y con ello al colonialismo chileno- es reafirmar la necesidad de establecerlo como un tema de derechos colectivos, en un contexto estatal donde conviven diversos pueblos. Dada la tradición republicana y constitucionalista del Estado chileno, el actual proceso constituyente no puede quedar fuera del análisis ni de la discusión política al interior del Pueblo Mapuche. Todo parece indicar que la Constitución de 1980 va a ser cambiada y la aprobación de un nuevo texto constitucional, si pretende cumplir con los estándares mínimos en lo que a pueblos indígenas se refiere, va a tener un reconocimiento constitucional de los pueblos indígenas. Sin embargo, la elevada expectativa generada al respecto en vastos sectores de la sociedad chilena, puede llevar a aprobar el actual proyecto de reconocimiento constitucional de los pueblos indígenas presentado en 2008 sin una consulta previa, el cual mantiene la impronta del multiculturalismo neoliberal predominante en las políticas públicas hacia pueblos indígenas en Chile y el continente durante la década de 1990, las cuales adolecen del reconocimiento de derechos políticos. De producirse esta situación, lo más seguro es que nos encontremos ante un nuevo intento fracasado para dar solución al conflicto, sumándose éste a una lista que tiene a su haber a la Ley indígena de 1993 y al nuevo trato del ex presidente Lagos, persistiendo el colonialismo en las relaciones chileno-mapuche.

\section{A modo de conclusión}

Como se indicó en un inicio, y siguiendo con los objetivos planteados, podemos concluir que el colonialismo ha sido una realidad con la que ha debido convivir el Pueblo Mapuche desde la instalación del régimen colonial español en Chile, el cual inaugura lo que las corrientes historiográficas chilenas han denominado periodo colonial. Esto puede sonar contradictorio con las propuestas historiográficas que, acertadamente, plantean que la resistencia militar mapuche logró detener, expulsar y fijar una frontera a la empresa de conquista hispana. Sin embargo, la novedad se encuentra en que, si bien esto es efectivo, no implica que el régimen colonial español haya desistido de la intención de extender sus dominios en el territorio mapuche, más bien lo que se produjo fue un aplazamiento del plan de conquista, debido al debilitamiento del régimen y la consecuente independencia chilena, heredando el nuevo régimen republicano el proyecto expansionista. La mantención de este proyecto durante todo el periodo colonial, implica que el Pueblo Mapuche no pudo estar ajeno a esta amenaza. De esta forma, la experiencia histórica del Pueblo Mapuche coincide con Fanon y su definición del origen del colonialismo como la imposición de la

\footnotetext{
${ }^{38}$ Pedro Cayuqueo, "Huenchumilla, yes we can", en Voces La Tercera, 13 de marzo de 2014,http://voces.latercera.com/2014/03/13/pedro-cayuqueo/huenchumilla-yes-we-can/ (Visitado el 4 de Junio de 2015)
} 
soberanía extranjera en un territorio conquistado, puesto que la conquista se termina por alcanzar con la operaciones militares planificadas por la República de Chile y que desarrollaron en el marco de la "Pacificación de la Araucanía" a fines del siglo XIX.

Siguiendo los lineamientos teóricos que ordenan las ideas expresadas a lo largo de este trabajo, también se puede concluir que desde la ocupación militar del territorio mapuche sus habitantes han vivenciado experiencias que pueden enmarcarse dentro de las expresiones que el colonialismo tiene en las relaciones sociales. Siguiendo a Spivak, se puede afirmar que el Pueblo Mapuche ha experimentado un proceso de subalternización dentro de la sociedad chilena, lo cual deriva en la imposibilidad de ejercer sus derechos como pueblo y en formas de discriminación de carácter racial y cultural que se originan en la construcción de la otredad como inferior. De ahí que existan, por ejemplo, una estratificación racializada del trabajo donde existen labores que en el imaginario de la sociedad chilena son considerados propios de los mapuche, como el trabajo de empleada doméstica o el de panadero.

Por último, es identificable una continuidad colonial en la historia del Pueblo Mapuche, pero ésta no puede reconocerse sin tomar en cuenta las reconfiguraciones que el colonialismo ha tenido a partir de la trayectoria histórica chilena, como señala Sergio Caniuqueo. En otras palabras, transformaciones tan trascendentales como la instalación del modelo neoliberal en la sociedad chilena colonizadora, sin duda que repercuten en una reconfiguración del colonialismo. Es así como llegamos a la actualidad, donde el colonialismo se presenta de forma más clara en el fracaso de las políticas de reconocimiento de derechos colectivos llevadas a cabo por los gobiernos que siguieron a la dictadura militar, graficadas en la inexistencia de participación real y efectiva de los mapuche en las decisiones que les incumben como pueblo, situación que tiene todas las posibilidades de repetirse en el proceso de cambio constitucional que se pretende llevar a cabo en Chile. De ser así, se habrá perdido una más de tantas posibilidades histórica que se han presentado para facilitar la disminución del carácter colonialista de la relación chilenomapuche.

Ahora bien, mientras persista la continuidad colonialista, también persistirán las propuestas descolonizadora -más allá de que una sea más viable que otra- en la medida que hayan sectores del Pueblo Mapuche que sigan pensando su realidad como la de un pueblo colonizado y desarrollando un proyecto autónomo, libre de cualquier tutelaje externo que impida retomar su soberanía suspendida. Mientras este proyecto no se concretice, la estrategia que sea la utilizada será un atenuante del colonialismo y no su fin, lo que mantendrá la necesidad de seguir investigando y sistematizando las experiencias descolonizadoras pasada, tanto propias como de otros pueblos y realidades colonizadas, para comprender las proyecciones del colonialismo como un orden sistémico. 
Con todo lo mencionado, no quedan dudas de que el caso mapuche es fiel reflejo de un pueblo colonizado, por más diversas que sean la interpretaciones que existan sobre el colonialismo, ya sea que se consideren las que se argumentan en el origen de la situación colonial (invasión que conduce a la perdida de la soberanía), así como también las que consideran sus múltiples consecuencia en las relaciones sociales (racismo, minorización, discriminación, etc). Es por esto que la consecución de los objetivos de este trabajo devino en un ejercicio metodológico con un alcance bidireccional: por un lado esclarecer las complejidades conceptuales que implica el concepto colonialismo a partir del caso mapuche, y al mismo tiempo, recoger dichos esclarecimiento para una relectura de la situación colonial del Pueblo Mapuche.

Recibido: 30 julio $2015 \quad$ Aceptado: 2 octubre 2015

\section{Bibliografía}

Alison Spedding, Descolonización. Crítica y problematización a partir del contexto boliviano, La paz, ISEAT. 2011

Aníbal Quijano, "Colonialidad del poder. Cultura y conocimiento en América Latina", Walter Mignolo (Comp.), Capitalismo y geopolítica del conocimiento. El eurocentrismo y la filosofía de la liberación en el debate intelectual contemporáneo, Buenos Aires, Ediciones del Signo, 2001, 117131.

"Colonialidad del poder y clasificación Social", Santiago Castro-Gómez y Ramón Grosfoguel, (comps.), Giro decolonial, Reflexiones para una diversidad epistémica más allá del capitalismo global, Bogotá, Siglo del Hombre Editores, 2007, 93 - 126.

Claudio Barrientos, Aproximaciones a la cuestión mapuche en Chile. Una mirada desde la historia y las ciencias sociales, Santiago de Chile,Ril Editores, 2014.

Edward Said, Orientalismo,Barcelona, Editorial Debate, 2002.

Enrique Antileo, "Frantz Fanon wallmapupüle. Apuntes sobre el colonialismo y posibilidades para repensar la nación en el caso mapuche", Elena Oliva; Lucia Stecher y Claudia Zapata (Eds.), Frantz Fanon desde América Latina. Lecturas contemporáneas de un pensador del siglo XX,Buenos Aires, Corregidor, 2012, 125 - 154.

"Trabajo racializado. Una reflexión a partir de datos de población indígena y testimonios de la migración y residencia mapuche en Santiago de Chile", Meridional. Revista chilena de estudios latinoamericanos, Número4, Santiago de Chile, 2015, 71-96. 
Frantz Fanon, Los condenados de la tierra, México, Fondo Cultura Económica, 2003.

,Piel negra, máscaras blancas, Buenos Aires, Editorial Abraxas, 1973.

Fernando Pairican, Malon. La rebelión del movimiento mapuche 1990-2013, Santiago de Chile, Pehuén Ediciones, 2014.

Gayatri Spivak, Crítica a la razón postcolonial. Hacia una historia del presente evanescente. Madrid, Ediciones Akal, 2010.

"Estudios de la subalternidad: deconstruyendo la historiografia", Silvia Rivera Cusicanqui y Rossana Barragán (Eds.),Debates Post Coloniales: Una introducción a los Estudios de la Subaltenidad. La Paz, SEPHIS- Editorial Historias, 1997, 247-278.

Grínor Rojo; Alicia Salomone y Claudia Zapata, "Postcolonialidad y nación: algunos aspectos de la discusión teórica”, Alejandra, Castillo et. al (Eds.) Nación, Estado y Cultura en América Latina, Santiago de Chile, Ediciones Facultad de Humanidades Universidad Chile, 2003, 17- 48.

Héctor Nahuelpan, "Las 'zonas grises' de las historias mapuche. Colonialismo internalizado, marginalidad y políticas de la memoria”, Revista de Historia Social y de las Mentalidades, Número 1, Volumen 17, Santiago de Chile, 2013, 9-31.

, "Formación colonial del Estado y desposesión en Ngulumapu", en Héctor Nahuelpan et. al., Ta iñ fijke xipa rakizuameluwün. Historia, colonialismo y resistencia desde el país Mapuche. Temuco, Ediciones Comunidad de Historia Mapuche, 2012, 119 - 152.

Jean Paul Sartre, Colonialismo y neocolonialismo. Situaciones V, Buenos Aires, Editorial Losada, 1965.

Jessabel Guamán Flores, "Pascua Lama y Ralco en la década de la emergencia indígena. Notas para una historia indígena comparada en Chile, 1990-2010", Historias que viven. Revista de estudiantes de Historia, Número 6, Santiago de Chile, 2015, 61- 83.

Jo SungHoon, Presentación en la $\mathrm{I}^{\circ}$ Jornadas Internacionales Intelectualidades Emergentes, Santiago de Chile, IDEA-USACH, 2014.

José Luis Cabrera Llancaqueo y Augusto Aillapan Paillafil, Machi mongen tani Santiago warria mew. Vida de un machi en la ciudad de Santiago, Santiago de Chile, Grupo de Trabajo Kuifike, 2013.

Jorge Vergara; Hans Gundermann, y Rolf Foertster, Estado, conflicto étnico y cultura. Estudios sobre pueblos indígenas en Chile, Antofagasta, Universidad Católica del Norte/QILLQA Universidad de Antofagasta, 2013. 
Luis G. de Mussy y Miguel Valderrama, Historiografía postmoderna. Conceptos, figuras, Manifiestos, Santiago de Chile, Ril Editores-Universidad Finis Terrae, 2010.

Miguel Valderrama, Epistemología de las ciencias sociales, Santiago de Chile, ARCIS-SELA, 2002.

Osvaldo Silva Galdames, Historia de Chile 2. La colonia 1601-1800, Santiago de Chile, Copesa, 2005.

Pedro Canales Tapia y Carmen Rea Campos, Claro de luz. Descolonización e "intelectualidades indigenas" en Abya Yala, siglos XX-XXI, Santiago de Chile, IDEA-USACH, 2013.

Pedro Cayuqueo, "Ecologistas de postal", en Mapuexpress, 14 de mayo de 2011. Disponible en:http://www.mapuexpress.net/content/publications/print.php?id=5046 (Visitado el 25 de mayo de 2015)

, "Huenchumilla, yes we can", en Voces La Tercera, 13 de marzo de 2014, disponible en:http://voces.latercera.com/2014/03/13/pedro-cayuqueo/huenchumilla-yes-we-can/(Visitado el 4 de Junio de 2015)

_, Solo por ser indios y otras crónicas mapuches, Santiago de Chile, Catalonia, 2012.

Ranajit Guha, "La prosa de contra insurgencia", Silvia Rivera Cusicanqui y Rossana Barragán (Eds.), Debates Post Coloniales. Una introducción a los Estudios de la Subaltenidad, La Paz, SEPHIS- Editorial Historias, 1997, 33 - 72.

Sergio Caniuqueo, "Siglo XX en Gulumapu: de la fragmentación del Wallmapu a la unidad nacional mapuche. 1880-1978", en Pablo Mariman et al..., Escucha wainka. Cuatro ensayos de historia nacional mapuche y un epílogo sobre el futuro", Santiago de Chile, Lom Ediciones, 2006, 129-217.

Tito Tricot, Autonomía. El Movimiento Mapuche de Resistencia. Santiago de Chile, Ceibo Ediciones, 2012.

Víctor Toledo, "Políticas Indígenas y Derechos Territoriales en América Latina: 1990-2004”, Pablo Dávalos, (Comp.),Pueblos indígenas y democracia en América latina. Buenos Aires: CLACSO, 2005, $67-102$.

, Pueblo Mapuche, derechos colectivos y territorio: desafios para la sustentabilidad democrática, Santiago de Chile, Programa Chile Sustentable, 2005.

Walter Mignolo, Capitalismo y geopolítica del conocimiento. El eurocentrismo y la filosofía de la liberación en el debate intelectual contemporáneo, Buenos Aires, Ediciones del Signo, 2001. 